\title{
Design and Analysis of Three Nonlinearly Activated ZNN Models for Solving Time-Varying Linear Matrix Inequalities in Finite Time
}

\author{
Yuejie Zeng ${ }^{1}$, Lin $\mathrm{XiaO}^{2 *}$, Kenli $\mathrm{Li}^{1 *}$, Jichun $\mathrm{Li}^{3}$, Keqin $\mathrm{Li}^{1}$, Zhen $\mathrm{Jian}^{1}$ \\ 1. College of Information Science and Engineering, Hunan University, Changsha \\ 410082, China. \\ 2. Hunan Provincial Key Laboratory of Intelligent Computing and Language Information \\ Processing, Hunan Normal University, Changsha, 410081, China. \\ 3. School of Science, Engineering and Design, Teesside University, Middlesbrough TS1 \\ 3BX, U.K.
}

\begin{abstract}
To obtain the superiority property of solving time-varying linear matrix inequalities (LMIs), three novel finite-time convergence zeroing neural network (FTCZNN) models are designed and analyzed in this paper. First, to make the Matlab toolbox calculation processing more conveniently, the matrix vectorization technique is used to transform matrix-valued FTCZNN models into vector-valued FTCZNN models. Then, considering the importance of nonlinear activation functions on the conventional zeroing neural network $(\mathrm{ZNN})$, the sign-bi-power activation function (AF), the improved sign-bipower AF and the tunable sign-bi-power AF are explored to establish the FTCZNN models. Theoretical analysis shows that the FTCZNN models not only can accelerate the convergence speed, but also can achieve finite-time convergence. Computer numerical results ulteriorly confirm the effectiveness and advantages of the FTCZNN models for finding the solution set of time-varying LMIs.
\end{abstract}

Keywords:

Zeroing neural network (ZNN), Time-varying linear matrix inequalities, Finite-time convergence, Vectorization technique, Sign-bi-power activation

\footnotetext{
*Corresponding author

Email addresses: xiaolin860728@163.com (Lin Xiao $\left.{ }^{2}\right), 1 k l @ h n u . e d u . c n\left(\mathrm{Kenli} \mathrm{Li}^{1}\right)$
} 


\section{Introduction}

For the past few years, as the inequalities grow in importance in various applications, the approaches based on solving inequalities are widely used to solve various problems in the engineering and science fields $[1,2,3,4,5]$. For example, in [6], a new way for solving inequalities which uses the projection neural network model was proposed. In [7], a method based on solving inequality was proposed and developed by Han et al., which was applied to non-fragile filtering for fuzzy systems. Besides, the linear matrix inequalities (LMIs) are viewed as an effective approach and design formulation, such as defining suitable LMIs to solve control design problems [8, 9]. In $[10,11,12,13]$, based on LMIs, they obtained the novel standards for asymptotic stability of Cohen-Grossberg neural networks, recurrent neural networks, delayed neural networks and delayed Hopfield neural networks, respectively. Furthermore, the global stability condition of neural networks is generally acquired by LMIs, such as Markovian jumping neural network [14], discrete recurrent neural network [15], discrete delayed impulsive interval neural network [16]. In addition, Liu et al. [17] realized the optimization of coupled neural network based on LMIs. In a word, solving LMIs has been a hot spot of research. Moreover, LMIs are widely used in practical application, such as obstacle avoidance for redundant robots [18, 19], motion scheme design based on physical limit avoidance [20,21], robot speed minimization [22] and robot manipulator control [23].

The conventional methods of solving LMIs are numerical algorithms that can solve the problem with special circumstances. For instance, under the condition that there is no analytic solution, the LMIs can be solved by convex optimization techniques [24]. Orsi et al. [25] came up with a novel method for solving LMIs based on alternating projection method, which controls the process of asymptotic convergence to analytic solution using dynamic systems. In order to accelerate the process of solving LMIs, Lin et al. [26] used a gradient neural network to solve LMIs for the first time. Compared with the traditional method, this method greatly reduced the solving time. In [27], Xiao et al. defined three zeroing neural network (ZNN) design formulas to solve LMIs. In [28, 29], Guo et al. presented a novel approach to solving LMIs by converting inequalities to equations. Syed Ali [30] presented a novel RNN based on LMIs to prove the global stability. 
However, the conventional algorithms and ZNN models [31] can not provide finite-time solution of LMIs. As is known that, Matlab toolbox can find the numerical solution of ordinary differential equations. Meanwhile, it can solve the linear inequalities by finding the upper bound of the solution set, such as $[32,33]$. At present, various recurrent neural network models were presented to solve equality problems with finite-time convergence. For instance, $\mathrm{Yu}$ et al. [34] put forward a novel activation function to achieve strong robustness and fixed time convergence for solving nonlinear equation. Matrix inversion was completed in finite time using the new ZNN model with sign-bi-power AF [35]. However, there is no work to design a finite-time recurrent neural network to solve time-varying LMIs. In this work, based on traditional ZNN models, three FTCZNN models are established by suggesting three different sign-bi-power AFs which can make the error functions of various neural network models converge to zero. The first one is the original sign-bi-power AF, the second one is a modification of the first one via amending sign-bi-power AF by adding a linear term, and the third one is activated by tunable sign-bi-power AF which defines three tunable parameters. Theoretical analysis shows that whatever the initial state is, the error function generated by the proposed FTCZNN models always converges to zero in finite time. Compared with the existing algorithms and conventional ZNN models, the main advantage of the proposed FTCZNN models lies in the fact that they can offer a faster rate and finite-time convergence property. The numerical simulation results verify the superiority of FTCZNN models.

The remainder of this paper is divided into four sections. In Section 2, the LMI problem is presented and the design method is given. Besides, the FTCZNN model vectorization has been done. Section 3 provides the convergence analysis. Section 4 shows the simulation results of three FTCZNN models. Section 5 is the conclusion of this paper. Before ending this part, it is necessary to point out the main contributions of this paper as below.

1) This paper proposes, researches and develops three novel FTCZNN models for solving LMI problems via applying three superior AFs. They achieve finite-time convergence for the online solution of LMI problems. This naturally becomes the most significant highlight of this work.

2) Three different nonlinear AFs are presented to establish FTCZNN models, which is via defining matrix-valued error functions, instead of the traditional scalar-valued energy functions. 
3) The theoretical analysis of the finite-time convergence guarantees the accuracy of the proposed FTCZNN models. By Lyapunov stability theory, the convergence upper bounds of FTCZNN models can be calculated precisely.

4) Numerical simulation results verify the superior convergence performance of three FTZCNN models for solving time-varying LMI problems, as compared with the existing ZNN models.

\section{FTCZNN Models}

In this section, some basic knowledge is presented at first. Then, based on the conventional ZNN model for LMIs, three FTCZNN models are designed by applying finite-time AFs.

\subsection{Problem Formulation}

In this part, the multi-dimensional time-varying LMI problem is formulated as below:

$$
A(t) X(t) B(t) \leq C(t),
$$

where $A(t) \in \mathbb{R}^{m \times m}, B(t) \in \mathbb{R}^{n \times n}$ and $C(t) \in \mathbb{R}^{m \times n}$ denote time-varying matrices. The purpose of this paper is to find the unknown solution set $X(t)$ which can make (1) hold true anytime.

\subsection{ZNN Model}

To monitor the process of solving LMIs, we define the matrix-vector error function as follows:

$$
E(t)=A(t) X(t) B(t)-C(t)
$$

where $E(t) \in \mathbb{R}^{m \times n}$.

Based on the efforts which have been done, the ZNN design formula for establishing the neural model is constructed as

$$
\dot{E}(t)=-\epsilon \operatorname{STP}(E(0)) \diamond \Psi(E(t)),
$$

where $\Psi(\cdot): \mathbb{R}^{m \times n} \rightarrow \mathbb{R}^{m \times n}$ is a monotonously increasingly odd activation function array, $\epsilon$ is a positive parameter and $\operatorname{STP}(\cdot): \mathbb{R}^{n} \rightarrow \mathbb{R}^{n}$ stands for a set of step functions which is defined as

$$
\operatorname{stp}(a)= \begin{cases}1, & a>0 \\ 0, & a \leq 0\end{cases}
$$


Besides, the operator $\diamond$ is defined as

$$
b \diamond c=\left[\begin{array}{c}
b_{1} c_{1} \\
b_{2} c_{2} \\
\vdots \\
b_{n} c_{n}
\end{array}\right]
$$

Note that the above ZNN design formula can make the error function converge to zero exponentially. Substituting (2) into (3), we have its implicit dynamic equation of the ZNN model:

$$
\begin{aligned}
A(t) \dot{X}(t) B(t)= & -\epsilon \operatorname{STP}(A(0) X(0) B(0)-C(0)) \diamond \Psi(A(t) X(t) B(t)-C(t)) \\
& -\dot{A}(t) X(t) B(t)-A(t) X(t) \dot{B}(t)+\dot{C}(t) .
\end{aligned}
$$

\subsection{ZNN Model Vectorization}

According to the implicit dynamic equation of ZNN model (6), $X(t)$ can not be calculated directly in Matlab. To address this problem, the Kronecker product is used to convert ZNN model (6) from the matrix form to the vector form. Then, ZNN model (6) is transformed into the following vector-valued one:

$$
\begin{aligned}
P(t) \dot{y}(t)= & -\epsilon \operatorname{STP}(P(0) y(0)-\operatorname{vec}(C(0))) \diamond \Psi(P(t) Y(t)-\operatorname{vec}(C(t)) \\
& -Q(t) y(t)-R(t) y(t)+\operatorname{vec}(\dot{C}(t))
\end{aligned}
$$

where $P(t)=B^{\mathrm{T}}(t) \otimes A(t)$ with $P(0)=B^{\mathrm{T}}(0) \otimes A(0), Q(t)=B^{\mathrm{T}}(t) \otimes \dot{A}(t)$, $R(t)=\dot{B}^{\mathrm{T}}(t) \otimes A(t)$ and $y(t)=\operatorname{vec}(X(t))$ with $y(0)=\operatorname{vec}(X(0))$, where $\operatorname{vec}(X(t))$ and $\operatorname{vec}(\dot{C}(t))$ denote the vectorization of $X(t)$ and $\operatorname{vec}(\dot{C}(t))$, respectively. In the following part, three finite-time convergent AFs are explored to shorten the convergence time and it is the first application to FTCZNN models for solving time-varying LMIs.

\subsection{Activation functions}

In the past years, the following AFs are widely applied in ZNN models:

1) the linear activation function:

$$
\Psi(x)=x
$$


2) the power activation function:

$$
\Psi(x)=x^{p}, p \geq 3
$$

3) the bipolar-sigmoid activation function:

$$
\Psi(x)=\frac{1+\exp (-p)}{1-\exp (-p)} \frac{1-\exp (-p x)}{1+\exp (-p x)}, p>2 ;
$$

4) the power-sigmoid activation function:

$$
\Psi(x)=\frac{1}{2} x^{p_{1}}+\frac{1+\exp \left(-p_{2}\right)}{1-\exp \left(-p_{2}\right)} \frac{1-\exp \left(-p_{2} x\right)}{1+\exp \left(-p_{2} x\right)}, p_{1} \geq 3, p_{2}>2 .
$$

However, the above mentioned AFs only can make the error function of ZNN model (6) converge to zero, and they cannot guarantee the finite convergence time in solving time-varying LMIs. To solve this problem, three superior finite-time AFs are applied to ZNN model (6), and the corresponding FTCZNN models are thus derived.

Specifically, the fist sign-bi-power AF is given as follows:

$$
\Psi_{1}(x)=\frac{1}{2}|x|^{r} \operatorname{sgn}(x)+\frac{1}{2}|x|^{\frac{1}{r}} \operatorname{sgn}(x),
$$

where $0<r<1$ and $\operatorname{sgn}(\cdot)$ is defined as

$$
\operatorname{sgn}(x)= \begin{cases}1, & \text { if } x>0 \\ 0, & \text { if } x=0 \\ -1, & \text { if } x<0\end{cases}
$$

In order to accelerate convergence, on basis of sign-bi-power AF (12), an improved sign-bi-power AF is designed by adding a linear term, and is presented as below:

$$
\Psi_{2}(x)=\frac{1}{2}|x|^{r} \operatorname{sgn}(x)+\frac{1}{2} x+\frac{1}{2}|x|^{\frac{1}{r}} \operatorname{sgn}(x) .
$$

To further reduce the theoretical convergence time upper bound, a tunable sign-bi-power AF is presented as below:

$$
\Psi_{3}(x)=\frac{1}{2} k_{1}|x|^{r} \operatorname{sgn}(x)+\frac{1}{2} k_{2} x+\frac{1}{2} k_{3}|x|^{\frac{1}{r}} \operatorname{sgn}(x) .
$$




\subsection{FTCZNN models}

In this section, by applying AFs (12), (14) and (15) to ZNN model (6), we can obtain the corresponding three FTCZNN models to solve time-varying LMIs. Their respective design processes of FTCZNN models are presented as below.

1) FTCZNN-1 model: Similar to the design process of (6), we can obtain the same error function. Then, the differential formula for this error function is shown as

$$
\dot{E}(t)=-\epsilon \operatorname{STP}(E(0)) \diamond \Psi_{1}(E(t)),
$$

where $\Psi_{1}(\cdot)$ denotes the sign-bi-power AF (12), $E(0)$ denotes the initial error of $E(t)$ at $t=0, \operatorname{STP}(\cdot)$ and $\diamond$ are defined as before.

At last, expanding the differential formula (16) by substituting $E(t)$, the dynamic equation corresponding to the FTCZNN-1 model is formed by

$$
\begin{aligned}
P(t) \dot{y}(t)= & -\epsilon \operatorname{STP}(P(0) y(0)-\operatorname{vec}(C(0))) \diamond \Psi_{1}(P(t) y(t)-\operatorname{vec}(C(t)) \\
& -Q(t) y(t)-R(t) y(t)+\operatorname{vec}(\dot{C}(t))
\end{aligned}
$$

where $P(t)=B^{\mathrm{T}}(t) \otimes A(t), Q(t)=B^{\mathrm{T}}(t) \otimes \dot{A}(t), R(t)=\dot{B}^{\mathrm{T}}(t) \otimes A(t)$, and $y(t)=\operatorname{vec}(X(t))$.

2) FTCZNN-2 model: On the basis of FTCZNN-1 model (17), we change the activation function to the improved sign-bi-power AF (14). Then, the differential formula for the error function is obtained as follows:

$$
\dot{E}(t)=-\epsilon \operatorname{STP}(E(0)) \diamond \Psi_{2}(E(t)),
$$

and the corresponding FTCZNN-2 model is formed by

$$
\begin{aligned}
P(t) \dot{y}(t)= & -\epsilon \operatorname{STP}(P(0) y(0)-\operatorname{vec}(C(0))) \diamond \Psi_{2}(P(t) y(t)-\operatorname{vec}(C(t)) \\
& -Q(t) y(t)-R(t) y(t)+\operatorname{vec}(\dot{C}(t)) .
\end{aligned}
$$

3) FTCZNN-3 model: On the foundation of FTCZNN-2 model (19), adding the tunable parameters to shorten convergence time, the differential formula for the error function is indicated below:

$$
\dot{E}(t)=-\epsilon \operatorname{STP}(E(0)) \diamond \Psi_{3}(E(t)) .
$$

Then, expanding the above equation, we get the following FTCZNN-3 model:

$$
\begin{aligned}
P(t) \dot{y}(t)= & -\epsilon \operatorname{STP}(P(0) y(0)-\operatorname{vec}(C(0))) \diamond \Psi_{3}(P(t) y(t)-\operatorname{vec}(C(t)) \\
& -Q(t) y(t)-R(t) y(t)+\operatorname{vec}(\dot{C}(t)) .
\end{aligned}
$$




\section{Theoretical Analysis}

In this part, we theoretically substantiate the convergent property of the proposed three FTCZNN models for solving LMIs. In addition, the finitetime convergence performance of FTCZNN models will be proved, with specific upper bound estimated. It is worth mentioning that when the initial state $X(0)$ is inside the solution set, we have $E(0)=A(0) X(0) B(0)-C(0) \leq$ 0 . That is, as the time $t$ goes by, $X(t)$ always stay in the solution set. Thus, in the following proofs, we only need to consider the situation when the initial state $X(0)$ is outside the solution set.

\subsection{Global Convergence}

It is the primary goal that we have to demonstrate the global convergence of the proposed three FTCZNN models which are activated by three different sign-bi-power activation functions.

Theorem 1: Given smoothly time-varying coefficient matrices $A(t) \in$ $\mathbb{R}^{m \times m}, B(t) \in \mathbb{R}^{n \times n}$ and $C(t) \in \mathbb{R}^{m \times n}$, FTCZNN-1 model (17), FTCZNN-2 model (19) and FTCZNN-3 model (21) achieve the global convergence.

Proof: According to the definitions of the novel AFs, we have

$$
\begin{gathered}
\Psi_{1}(-x)=\frac{1}{2}|-x|^{r} \operatorname{sgn}(-x)+\frac{1}{2}|-x|^{\frac{1}{r}} \operatorname{sgn}(-x) \\
=-\frac{1}{2}|x|^{r} \operatorname{sgn}(x)-\frac{1}{2}|x|^{\frac{1}{r}} \operatorname{sgn}(x) \\
=-\Psi_{1}(x) ; \\
\Psi_{2}(-x)=\frac{1}{2}|-x|^{r} \operatorname{sgn}(-x)-\frac{1}{2} x+\frac{1}{2}|-x|^{\frac{1}{r}} \operatorname{sgn}(-x) \\
=-\frac{1}{2}|x|^{r} \operatorname{sgn}(x)-\frac{1}{2} x-\frac{1}{2}|x|^{\frac{1}{r}} \operatorname{sgn}(x) \\
=-\Psi_{2}(x) ; \\
\Psi_{3}(-x)=\frac{1}{2} k_{1}|-x|^{r} \operatorname{sgn}(-x)-\frac{1}{2} k_{2} x+\frac{1}{2} k_{3}|-x|^{\frac{1}{r}} \operatorname{sgn}(-x) \\
=-\frac{1}{2} k_{1}|x|^{r} \operatorname{sgn}(x)-\frac{1}{2} k_{2} x-\frac{1}{2} k_{3}|x|^{\frac{1}{r}} \operatorname{sgn}(x) \\
=-\Psi_{3}(x) .
\end{gathered}
$$

Therefore, we can know that the three AFs are monotonically increasing odd functions. 
Then, let us define a Lyapunov function $u_{i j}(t)=\varrho_{i j}^{2}(t) / 2$, where $\varrho_{i j}(t)$ is the element of $E(t)$ which is defined in (2). Since $X(0)$ is outside the solution set, $\operatorname{STP}(E(0))=1$ which means that every element of $\operatorname{STP}(E(0))$ equals to 1. Therefore, we have $\operatorname{STP}\left(\varrho_{i j}(0)\right)=1$. Thus, $\dot{u}_{i j}(t)$ is computed as

$$
\dot{u}_{i j}(t)=\frac{\mathrm{d} u_{i j}(t)}{\mathrm{d} t}=-\epsilon \varrho_{i j}(t) \dot{\varrho}_{i j}(t)=-\epsilon \varrho_{i j}(t) \Psi_{k}\left(\varrho_{i j}(t)\right), \quad k=1,2,3 .
$$

As shown in the above, $\Psi_{k}(\cdot)$ is monotonically increasing, so we obtain

$$
\varrho_{i j}(t) \Psi_{k}\left(\varrho_{i j}(t)\right) \begin{cases}>0, & \text { if } \varrho_{i j}(t) \neq 0 ; \\ =0, & \text { if } \varrho_{i j}(t)=0 ;\end{cases}
$$

which guarantees that $\dot{u}_{i j}(t)<0$ for $\varrho_{i j}(t) \neq 0$, and $u_{i j}(t)=0$ for $\varrho_{i j}(t)=0$. That is to say, $\varrho_{i j}(t) \leq 0$ for any $i, j$, so $E(t)$ can converge to zero.

The proof is completed.

\subsection{Finite-Time Convergence Analysis}

The three FTCZNN models not only can achieve global convergence but also can accomplish the finite-time convergence. In another word, they have better convergence performance. In this section, we will provide three theorems to show the finite-time convergent property of the proposed three FTCZNN models.

Theorem 2: Given smoothly time-varying coefficient matrices $A(t) \in$ $\mathbb{R}^{m \times m}, B(t) \in \mathbb{R}^{n \times n}$ and $C(t) \in \mathbb{R}^{m \times n}$, FTCZNN-1 model (17) can achieve finite-time convergence. The convergence time upper bound $T_{1}$ satisfies the following equation:

$$
T_{1} \leq \begin{cases}\frac{2 r\left(L(0)^{(r-1) / 2 r}-1\right)}{\epsilon(r-1)}+\frac{2}{\epsilon(1-r)}, & L(0) \geq 1, \\ \frac{2}{\epsilon(1-r)} L(0)^{(1-r) / 2}, & L(0)<1,\end{cases}
$$

where $L(0)=\left|\varrho^{+}(0)\right|^{2}$ with $\varrho^{+}(0)=\max \left\{\left|\varrho_{i j}(0)\right|\right\}$.

Proof: When $X(0)$ is outside the solution set, $\operatorname{STP}(E(0))=1$ which means that every element of $\operatorname{STP}(E(0))$ equals to 1 . Therefore, we have $\operatorname{STP}\left(\varrho_{i j}(0)\right)=1$. From (16), we acquire

$$
\dot{\varrho}_{i j}(t)=-\epsilon \Psi_{1}\left(\varrho_{i j}(t)\right), i=1,2, \cdots, n .
$$

Then, we define $\varrho^{+}(t)=\max \left|\left(\varrho_{i j}(t)\right)\right|$ which is used to calculate the convergence time upper bound. By Comparison Lemma, we have $-\left|\varrho^{+}(t)\right|<$ 
$\varrho_{i j}(t)<\left|\varrho^{+}(t)\right|$. Hence, we just need guarantee that $\left|\varrho^{+}(t)\right|$ achieves finitetime convergence. Substituting the expression of $\left|\varrho^{+}(t)\right|$ into (28), we have

$$
\dot{\varrho}^{+}(t)=-\epsilon \Psi_{1}\left(\varrho^{+}(t)\right) .
$$

Define the Lyapunov function $L(t)=\left|\varrho^{+}(t)\right|^{2}$, whose time derivative along this dynamics is computed as follows:

$$
\begin{aligned}
\dot{L}(t) & =2 \varrho^{+}(t) \dot{\varrho}^{+}(t) \\
& =-2 \epsilon \varrho^{+}(t) \Psi_{1}\left(\varrho^{+}(t)\right) \\
& =-\epsilon\left(\left|\varrho^{+}(t)\right|^{r+1}+\left|\varrho^{+}(t)\right|^{\frac{1}{r}+1}\right) \\
& =-\epsilon\left(L^{\frac{r+1}{2}}+L^{\frac{r+1}{2 r}}\right) .
\end{aligned}
$$

If $L(0) \geq 1$, from the equation (29), we have the following inequality:

$$
\dot{L} \leq-\epsilon L^{\frac{r+1}{2 r}}
$$

from which we can obtain

$$
\mathrm{d} L \leq-\epsilon L^{\frac{r+1}{2 r}} \mathrm{~d} t
$$

Integrating both sides of the formula (31) from 0 to $t$, we have

$$
\int_{L(0)}^{L(t)} L^{-\frac{r+1}{2 r}} \mathrm{~d} L \leq-\epsilon \int_{0}^{t} \mathrm{~d} t .
$$

Simplifying the inequality after integration yields to

$$
L(t) \leq\left[\frac{r-1}{2 r}\left(-\epsilon t+\frac{2 r}{r-1} L(0)^{\frac{r-1}{2 r}}\right)\right]^{\frac{2 r}{r-1}} .
$$

Setting the left-hand side of this inequality equal to 1 , we get the value of $t_{1}$ :

$$
t_{1}=\frac{2 r\left(L(0)^{(r-1) / 2 r}-1\right)}{\epsilon(r-1)} .
$$

Thus, after time $t_{1}, L(t)$ decreases to 1 . When $L(t) \leq 1$, the inequality (29) shows that

$$
\dot{L} \leq-\epsilon L^{\frac{r+1}{2}}
$$


Similar to solving for $t_{1}$, we compute the remaining convergence time $t_{2}$ :

$$
t_{2}=\frac{2}{(1-r) \epsilon}
$$

Hence, we obtain the convergence time upper bound $T_{1}<t_{1}+t_{2}$.

If $L(0) \leq 1$, from the equation (29), we have the following inequality:

$$
\dot{L} \leq-\epsilon L^{\frac{r+1}{2}}, \text { and } \mathrm{d} L \leq-\epsilon L^{\frac{r+1}{2}} \mathrm{~d} t .
$$

Integrating both sides of the differential inequality $\int_{L(0)}^{L(t)} L^{-\frac{r+1}{2}} \mathrm{~d} L \leq-\epsilon \int_{0}^{t} \mathrm{~d} t$, the convergence time upper bound $t_{3}$ can be computed as

$$
t_{3}=\frac{2}{\epsilon(1-r)} L(0)^{\frac{(1-r)}{2}} .
$$

The proof is completed.

Theorem 3: Given smoothly time-varying coefficient matrices $A(t) \in$ $\mathbb{R}^{m \times m}, B(t) \in \mathbb{R}^{n \times n}$ and $C(t) \in \mathbb{R}^{m \times n}$, FTCZNN-2 model (19) can accomplish finite-time convergence for solving time-varying LMIs. The convergence time upper bound $T_{2}$ is calculated as

$$
T_{2} \leq \begin{cases}\frac{2 r \ln \left[\frac{2}{L(0)^{(r-1) / 2 r}+1}\right]}{\epsilon(1-r)}+\frac{2 \ln 2}{\epsilon(1-r)}, & L(0) \geq 1, \\ \frac{2 \ln \left[1+L(0)^{(1-r) / 2}\right]}{\epsilon(1-r)}, & L(0)<1,\end{cases}
$$

where $r, \epsilon$ and $L(0)$ are defined as before.

Proof: Similar to Theorem 2, from (18), we have

$$
\dot{\varrho}_{i j}(t)=-\epsilon \Psi_{2}\left(\varrho_{i j}(t)\right), i=1,2, \cdots, n .
$$

Then, the derivative of the Lyapunov function along time $t$ is computed as follows:

$$
\begin{aligned}
\dot{L} & =2 \varrho^{+}(t) \dot{\varrho}^{+}(t) \\
& =-2 \epsilon \varrho^{+}(t) \Psi_{2}\left(\varrho^{+}(t)\right) \\
& =-\epsilon\left(\left|\varrho^{+}(t)\right|^{r+1}+\left|\varrho^{+}(t)\right|^{2}+\left|\varrho^{+}(t)\right|^{\frac{1}{r}+1}\right) \\
& =-\epsilon\left(L^{\frac{r+1}{2}}+L+L^{\frac{r+1}{2 r}}\right) .
\end{aligned}
$$

If $L(0) \geq 1$, considering the equation (39), the following inequality is satisfied:

$$
\dot{L} \leq-\epsilon\left(L+L^{\frac{r+1}{2 r}}\right),
$$


which can be rewritten as

$$
\frac{\mathrm{d} L}{L^{(r+1) / 2 r}+L} \leq-\epsilon \mathrm{d} t .
$$

Integrating the formula (41) from 0 to $t$ yields

$$
\int_{L(0)}^{L(t)} \frac{1}{L^{(r+1) / 2 r}+L} \mathrm{~d} L \leq \int_{0}^{t}-\epsilon \mathrm{d} t,
$$

which can be rewritten as follows:

$$
\frac{2 r}{r-1} \int_{L(0)}^{L(t)} \frac{1}{1+L^{(r-1) / 2 r}} \mathrm{~d}\left(L^{(r-1) / 2 r}\right) \leq \int_{0}^{t}-\epsilon \mathrm{d} t .
$$

Let $L(t)$ equal to $1, t_{4}$ satisfies the following equality:

$$
t_{4}=\frac{2 r \ln \left[\frac{2}{L(0)^{(r-1) / 2 r}+1}\right]}{\epsilon(1-r)} .
$$

When $t \geq t_{4}$, we have $L(t) \leq 1$. It follows from the condition (39) that

$$
\dot{L} \leq-\epsilon\left(L^{\frac{r+1}{2}}+L\right) \text {. }
$$

There exists $t_{5}$ satisfying the equation:

$$
t_{5}=\frac{2 \ln 2}{\epsilon(1-r)}
$$

The convergence time upper bound $T_{2}<t_{4}+t_{5}$.

If $L(0) \leq 1$, the inequality (43) holds and its differential form can be obtained:

$$
\frac{\mathrm{d} L}{L+L^{(r+1) / 2}} \leq-\epsilon \mathrm{d} t
$$

Integrating both sides of the formula from 0 to $t$, we have

$$
\int_{L(0)}^{L(t)} \frac{1}{L+L^{(r+1) / 2}} \mathrm{~d} L \leq \int_{0}^{t}-\epsilon \mathrm{d} t
$$

which can be rewritten as

$$
\frac{2}{1-r} \int_{L(0)}^{L(t)} \frac{1}{1+L^{(1-r) / 2}} \mathrm{~d}\left(L^{(1-r) / 2}\right) \leq \int_{0}^{t}-\epsilon \mathrm{d} t .
$$


Thus, the convergence upper bound $T_{2}<t_{6}$ satisfies the following equation:

$$
t_{6}=\frac{2 \ln \left[1+L(0)^{(1-r) / 2}\right]}{\epsilon(1-r)} .
$$

That completes the proof.

Theorem 4: Given smoothly time-varying coefficient matrices $A(t) \in$ $\mathbb{R}^{m \times m}, B(t) \in \mathbb{R}^{n \times n}$ and $C(t) \in \mathbb{R}^{m \times n}$, FTCZNN-3 model (21) can accomplish finite-time convergence and greatly shorten the convergence time. Its upper bound $T_{3}$ satisfies the following formula:

$$
T_{4} \leq \begin{cases}\frac{2 r \ln \left[\frac{1+\frac{k_{2}}{k_{3}} L(0)^{(r-1) / 2 r}}{1+\frac{k_{2}}{k_{3}}}\right]}{k_{2} \epsilon(r-1)}+\frac{2 \ln \left[1+\frac{k_{2}}{k_{1}}\right]}{k_{2} \epsilon(1-r)}, & L(0) \geq 1, \\ \frac{2 \ln \left[1+\frac{k_{2}}{k_{1}} L(0)^{(1-r) / 2}\right]}{k_{2} \epsilon(1-r)}, & L(0)<1,\end{cases}
$$

where $r, \epsilon$ and $L(0)$ are defined as before.

Proof: From (20), the $i j$ th element of $E(t)$ can be rewritten as

$$
\dot{\varrho}_{i j}(t)=-\epsilon \Psi_{3}\left(\varrho_{i j}(t)\right) .
$$

According to the definition of the Lyapunov function as before, its derivative that reflects the dynamic change of the model is obtained as

$$
\begin{aligned}
\dot{L} & =2 \varrho^{+}(t) \dot{\varrho}^{+}(t) \\
& =-2 \epsilon \varrho^{+}(t) \Psi_{3}\left(\varrho^{+}(t)\right) \\
& =-\epsilon\left(k_{1}\left|\varrho^{+}(t)\right|^{r+1}+k_{2}\left|\varrho^{+}(t)\right|^{2}+k_{3}\left|\varrho^{+}(t)\right|^{\frac{1}{r}+1}\right) \\
& =-\epsilon\left(L^{k_{1} \frac{r+1}{2}}+k_{2} L+k_{3} L^{\frac{r+1}{2 r}}\right) .
\end{aligned}
$$

If $L(0)>1$, the following result is satisfied:

$$
\dot{L} \leq-\epsilon\left(k_{2} L+k_{3} L^{\frac{r+1}{2 r}}\right),
$$

which can be written as

$$
\frac{2 r}{k_{3}(r-1)} \cdot \frac{\mathrm{d}\left(L^{(r-1) / 2 r)}\right.}{\left.1+\frac{k_{2}}{k_{3}} L^{(}(r-1) / 2 r\right)} \leq-\epsilon t .
$$

Integrating two side of (51) from 0 to $t$,

$$
\frac{2 r}{k_{2}(r-1)} \int_{L(0)}^{L(t)} \frac{1}{\left.1+\frac{k_{2}}{k_{3}} L^{(}(r-1) / 2 r\right)} \mathrm{d}\left(L^{(r-1) / 2 r}\right) \leq \int_{0}^{t}-\epsilon \mathrm{d} t .
$$


Let $L(t)$ equal to 1 , we can get the time $t_{7}$ :

$$
t_{7}=\frac{2 r}{k_{2} \epsilon(1-r)} \ln \left[\frac{1+\frac{k_{2}}{k_{3}} L(0)^{(r-1) / 2 r}}{1+\frac{k_{2}}{k_{3}}}\right] .
$$

When $L(0)<1$, (49) satisfies the following equation:

$$
\dot{L} \leq-\epsilon\left(k_{1} L^{\frac{r+1}{2}}+k_{2} L\right) \text {. }
$$

Repeating this process until the $L(t)$ converges to 0 , we can get $t_{8}$ :

$$
t_{8}=\frac{2 \ln \left[1+\frac{k_{2}}{k_{1}}\right]}{k_{2} \epsilon(1-r)} \text {. }
$$

The upper bound of convergence time $T_{4}<t_{7}+t_{8}$.

If $L(0)<1$, analog to the proving course of $L(0)>1$, the derivative of Lyapunov function can be shown as

$$
\frac{2}{k_{2}(1-r)} \int_{L(0)}^{L(t)} \frac{1}{1+\frac{k_{2}}{k_{1}} L^{(1-r) / 2}} \mathrm{~d}\left(L^{(1-r) / 2}\right) \leq \int_{0}^{t}-\epsilon \mathrm{d} t .
$$

In the same way, integrating both side of the type, we can calculate the convergence time $t_{9}$ as

$$
t_{9}=\frac{2 \ln \left[1+\frac{k_{2}}{k_{1}} L(0)^{(1-r) / 2}\right]}{k_{2} \epsilon(1-r)} .
$$

This proof is completed.

\section{Simulative Verification}

In the previous two sections, three FTCZNN models together with their theoretical analysis have been presented. In this part, for illustration and comparison, a numerical example is given to evaluate the performance of the FTCZNN models activated by different AFs for solving time-varying LMIs. To testify the superiority of three FTCZNN models, some comparative experiments have also been conducted under the same conditions. 


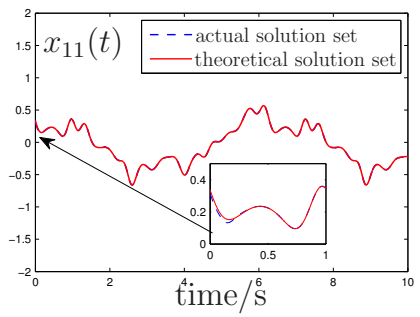

(a)

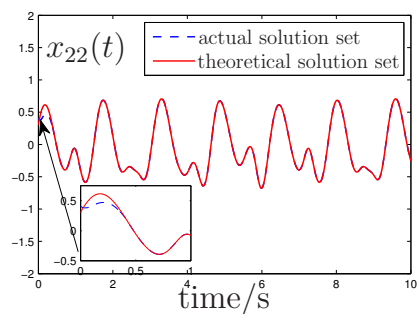

(d)

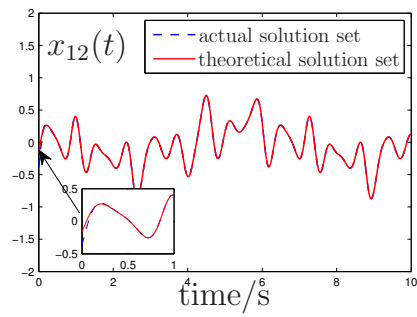

(b)

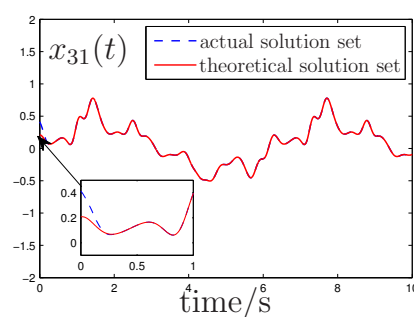

(e)

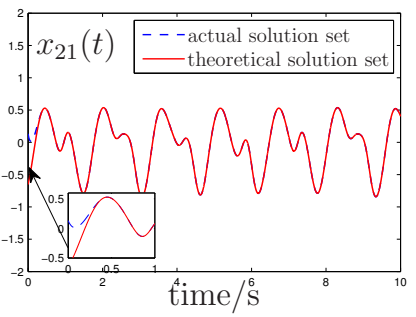

(c)

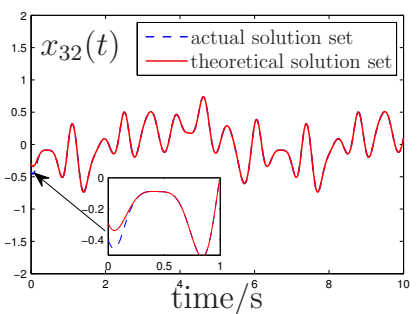

(f)

Figure 1: Trajectories of state vector $x(t)$ by applying FTCZNN-1 model (17) to solve LMI (1) when $x(0)$ is outside $\Omega(0)$ with $\epsilon=1$ and $r=0.3$. (a) $x_{11}(t)$ (b) $x_{12}(t)$ (c) $x_{21}(t)$ (d) $x_{22}(t)(\mathrm{e}) x_{31}(t)(\mathrm{f}) x_{32}(t)$

\subsection{Constant Parameter}

For illustration and simulation, let us consider a specific time-varying LMI with the following coefficient matrices $A(t), B(t)$ and $C(t)$ :

$$
\begin{gathered}
A(t)=\left[\begin{array}{ccc}
3+\sin (4 t) & \cos (4 t) / 2 & \cos (4 t) \\
\cos (4 t) / 2 & 3+\sin (4 t) & \cos (4 t) / 2 \\
\cos (4 t) & \cos (4 t) / 2 & 3+\sin (4 t)
\end{array}\right], \\
B(t)=\left[\begin{array}{cc}
\sin (4 t) & \cos (4 t) \\
-\cos (4 t) & \sin (4 t)
\end{array}\right] \text { and } \\
C(t)=\left[\begin{array}{cc}
\sin (3 t) & \cos (3 t) \\
\cos (4 t)+1 & \sin (4 t)+1 \\
\sin (5 t)+\cos (5 t) & \sin (5 t) \cos (5 t)
\end{array}\right] .
\end{gathered}
$$

Fig. 1 shows the state trajectories of $X(t)$ by applying FTCZNN-1 model (17) with $\epsilon=1$ and $r=0.5$, where the red solid lines represent the theoretical upper bound of the solution set with each element denoted by $x_{i j}^{*}(t)$, while the blue dotted lines show the actual trajectories with each element denoted by $x_{i j}(t)$. It can be seen that the blue dotted line gradually coincides with 


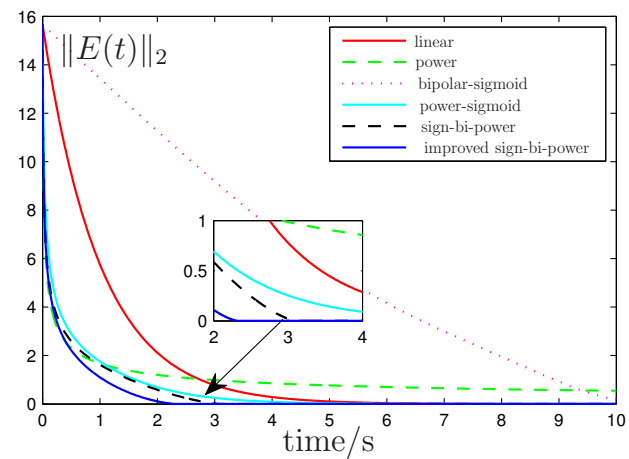

(a)

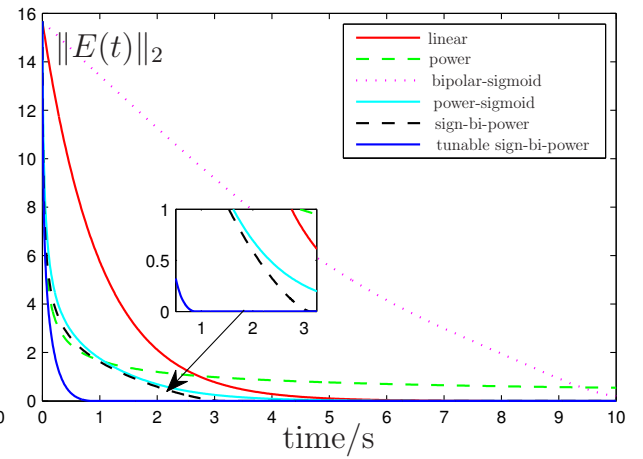

(b)

Figure 2: Comparisons of three FTCZNN models with the conventional ZNN models activated by other AFs with $\epsilon=1$ and $r=0.3$ when $L(0)>1$. (a) By FTCZNN-1 model (17) and FTCZNN-2 model (19). (b) By FTCZNN-1 model (17) and FTCZNN-3 model (21).

the red one over time. In other words, the simulation results shown in Fig. 1 (a)-(f) can constitute the solution of the above time-varying LMI.

Then, the residual errors $\|P(t) y(t)-\operatorname{vec}(C(t))\|_{2}=\| A(t) X(t) B(t)-$ $C(t) \|_{\mathrm{F}}$ (where $\|\cdot\|_{2}$ denotes two norm of a vector and $\|\cdot\|_{\mathrm{F}}$ denotes Frobenius norm of a matrix) are shown in Fig. 2. Obviously, the residual error function reflects the whole convergence process. From Fig. 2, three FTCZNN models obviously have shorter convergence time than the conventional ZNN models activated by the linear activation function (8), the power function (9), the bipolar-sigmoid function (10) and the smooth power-sigmoid function (11) [40, 41, 42, 43]. Moreover, among them, FTCZNN-3 model (21) with three tunable parameters obtains the best convergence performance for solving LMIs. In addition, it can be seen that such three FTCZNN models can reach zero within $3 \mathrm{~s}$. Meanwhile, the corresponding results of the traditional ZNN models for finding LMIs solution still have some estimation errors at this time.

For the sake of demonstrating the finite-time convergence property of FTCZNN models for solving LMIs, a specific initial state $X(0)$ is given. According to the preceding theorems, we can calculate the convergence time upper bound. Considering that different states can lead to different convergence upper bound, the simulation cases are decomposed into two parts.

Case I Let $X(0)=[-1,-0.5 ;-3,2 ;-1.5,1]$, which represents that $A(0) X(0) B(0)-C(0)=[-0.5,-7 ;-8.25,-11.25 ;-4.5,-7]$, and $L(0)=$ 


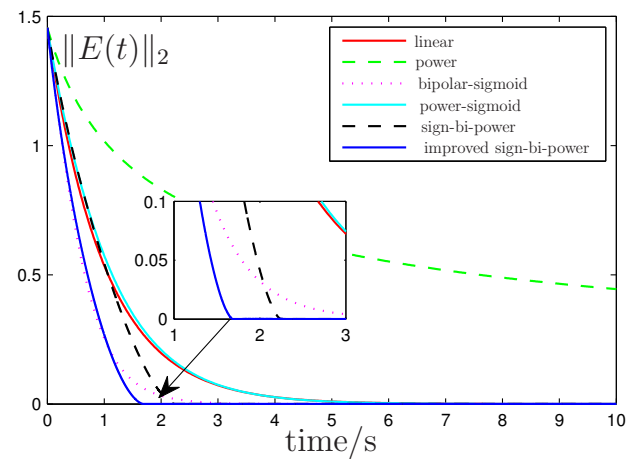

(a)

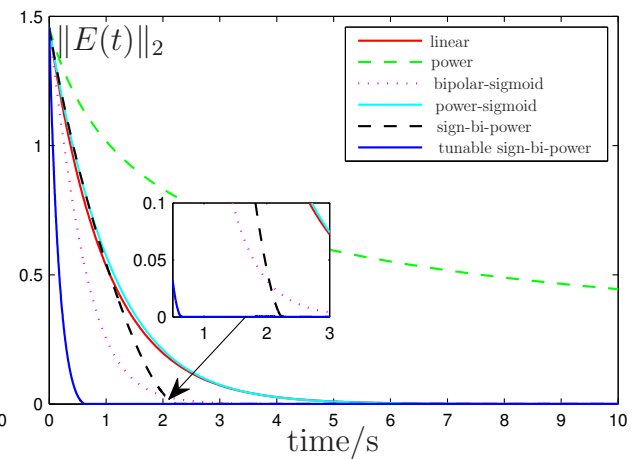

(b)

Figure 3: Comparisons of three FTCZNN models with the conventional ZNN models activated by other AFs with $\epsilon=1$ and $r=0.3$ when $L(0)<1$. (a) By FTCZNN-1 model (17) and FTCZNN-2 model (19). (b) By FTCZNN-1 model (17) and FTCZNN-3 model (21).

$\left|\varrho^{+}(0)\right|^{2}=(11.25)^{2}=126.5625$. The parameters of FTCZNN-1 model (17) are given as $r=0.3$ and $\epsilon=1$. By Theorem 2, the convergence time upper bound $t_{a}$ is given as

$$
t_{a}=\frac{2 r\left(L(0)^{(r-1) / 2 r}-1\right)}{\epsilon(r-1)}+\frac{2}{\epsilon(1-r)} \approx 3.7113 \mathrm{~s} .
$$

Let $r=0.3$ and $\epsilon=1$. By Theorem 3, the convergence time upper bound $t_{b}$ for FTCZNN-2 model (19) is given as

$$
t_{b}=\frac{2 r \ln \left[\frac{2}{L(0)^{(r-1) / 2 r}+1}\right]}{\epsilon(1-r)}+\frac{2 \ln 2}{\epsilon(1-r)} \approx 2.3942 \mathrm{~s} .
$$

Keeping $r$ and $\epsilon$ the unchanged, let $k_{1}=1, k_{2}=10$, and $k_{3}=1$. According to Theorem 4, the convergence time upper bound $t_{c}$ for FTCZNN-3 model (21) is computed as

$$
t_{c}=\frac{2 r \ln \left[\frac{1+\frac{k_{2}}{k_{3}} L(0)^{(r-1) / 2 r}}{1+\frac{k_{2}}{k_{3}}}\right]}{k_{3} \epsilon(1-r)}+\frac{2 \ln \left[1+\frac{k_{2}}{k_{1}}\right]}{k_{1} \epsilon(1-r)} \approx 1.0227 \mathrm{~s} .
$$

From Fig. 2, it can be seen that the actual convergence time is smaller or equal to their corresponding theoretical upper bound of convergence time. 


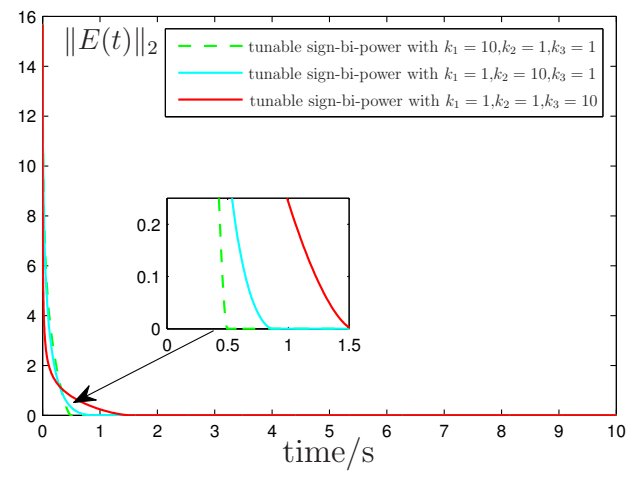

(a)

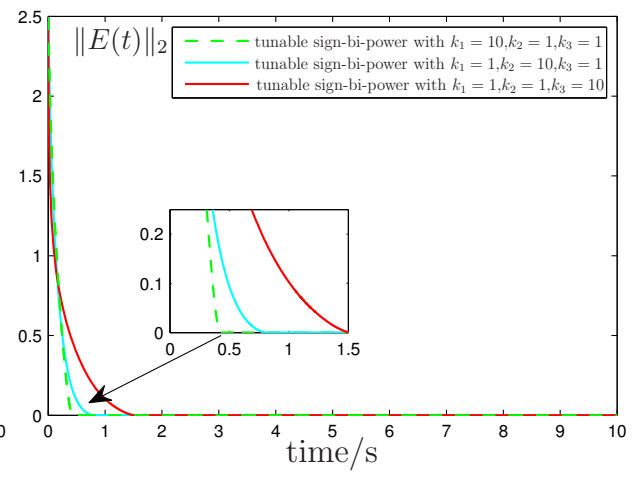

(b)

Figure 4: Comparisons among three cases of FTCZNN-3 model (21) with different tunable parameters. (a) $L(0)>1$. (b) $L(0)<1$.

Specifically, FTCZNN-3 model (21) for solving LMIs can converge to zero within 0.75 s, FTCZNN-2 model (19) spends $2.12 \mathrm{~s}$, and FTCZNN-1 model (17) needs $2.95 \mathrm{~s}$ to complete the process of convergence. Moreover, the distance between the actual time and theoretical analysis value can express the convergence performance. Hence, it can easily be concluded that FTCZNN-3 model (21) has the best convergence performance, followed by FTCZNN-2 model (19), and finally FTCZNN-1 model (17).

Case II Let $X(0)=[0.3,0.008 ; 0.1,-0.08 ;-0.34,-0.3]$, which represents that $A(0) X(0) B(0)-C(0)=[0.71,-0.79 ; 0.15,-0.004 ;-0.78,0.6]$, and $L(0)=\left|\varrho^{+}(0)\right|^{2}=(0.79)^{2}=0.6241<1$. The parameters of FTCZNN-1 model (17) are given as $r=0.3$ and $\epsilon=1$. By Theorem 2, the convergence time upper bound $t_{a}$ is given as

$$
t_{a}=\frac{2}{\epsilon(1-r)} L(0)^{(1-r) / 2} \approx 2.4225 \mathrm{~s} .
$$

While using FTCZNN-2 model (19) to solve (1), by Theorem 3, the convergence time upper bound $t_{b}$ is given as

$$
t_{b}=\frac{2 \ln \left[1+L(0)^{(1-r) / 2}\right]}{\epsilon(1-r)} \approx 1.7544 \mathrm{~s} .
$$

Let $k_{1}=1, k_{2}=10$, and $k_{3}=1$, by Theorem 4 , the convergence time upper 


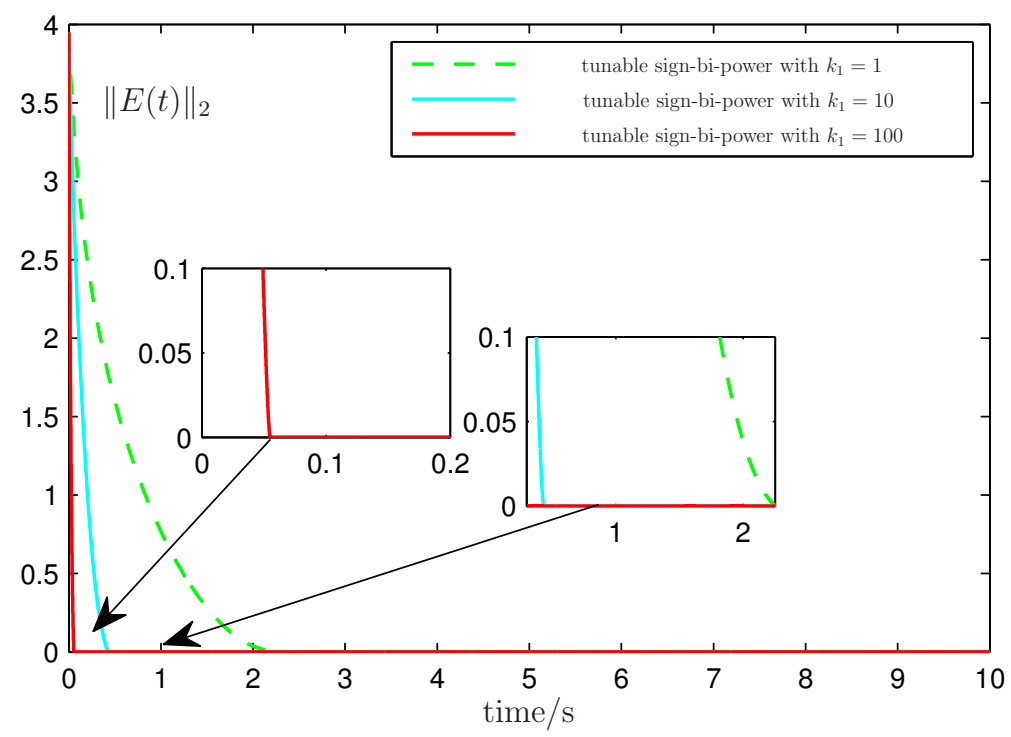

Figure 5: Transient behaviors of the error function $\|E(t)\|_{2}$ synthesized by FTCZNN-3 model (21) with $k_{2}=1, k_{3}=1$ and different values of $k_{1}$.

bound $t_{c}$ for FTCZNN-3 model (21) is calculated as

$$
t_{c}=\frac{2 \ln \left[1+\frac{k_{2}}{k_{1}} L(0)^{(1-r) / 2}\right]}{k_{2} \epsilon(1-r)} \approx 0.6426 \mathrm{~s} .
$$

Similar to the situation when $L(0)>1$, from Fig. 3, three FTCZNN models have better convergence performance. In addition, the distance between the theoretical computational time and the actual time using FTCZNN-3 model (21) is shorter than the other. It demonstrates the superiority of FTCZNN-3 model (21) with the tunable sign-bi-power AF via numerical simulations.

For further investigation, we have done a contrast experiment to determine which parameter has the greatest effect on the convergence rate of FTCZNN-3 model (21). In Fig. 4, the aforementioned LMI problem can be solved by setting three different cases for FTCZNN-3 model (21), i.e., $k_{1}=10, k_{2}=1$ and $k_{3}=1 ; k_{1}=1, k_{2}=10$ and $k_{3}=1 ;$ and $k_{1}=0, k_{2}=1$ and $k_{3}=10$. According to the simulation results, we can see that three cases are able to make the residual error achieve to zero rapidly. Under the first case, i.e., $k_{1}=10, k_{2}=1$ and $k_{3}=1$, the convergence time is least. This is consistent with the results of theoretical analysis. Because whatever the 


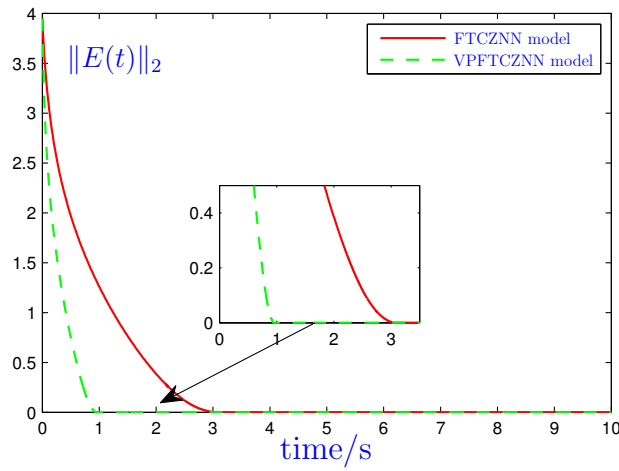

(a)

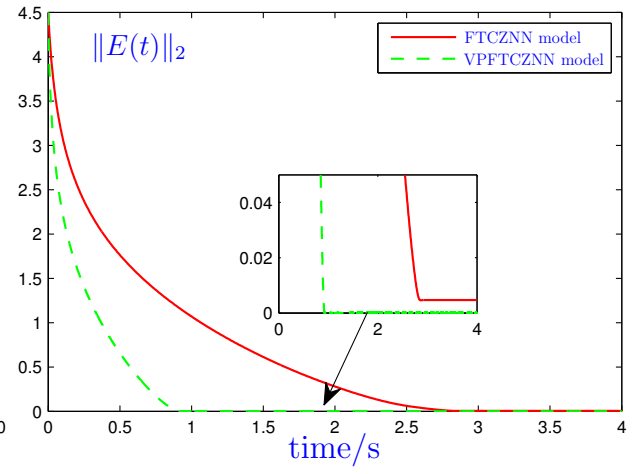

(b)

Figure 6: Comparisons between FTCZNN model (16) and the varying-parameter FTCZNN model (57) for solving time-varying linear matrix inequalities. (a) Free noise. (b) Random noise generated from $0.1 * \operatorname{rand}(6,1)$.

initial state $L(0)$ is, there is always a period when $L(0)<1$. In other words, the time from $L(0)<1$ to 0 is unavoidable and must be experienced. What determines the length of convergence time for FTCZNN-3 model (21) is the value of $k_{1}$, so the greater $k_{1}$, the shorter convergence time.

Furthermore, in Fig. 5, it is worth pointing out that the convergence time reduced from 2.17 seconds to 0.425 seconds when the value of $k_{1}$ is increased from 1 to10. When $k_{1}=100$, the residual error only needs 0.055 seconds to converge to zero. Therefore, the value of $k_{1}$ is an important influential factor for the convergence time of FTCZNN-3 model (21) when applied to solving time-varying LMIs.

\subsection{Varying Parameter FTCZNN Model}

Inspired by [36, 37, 38, 39], the varying parameters have been considered in the traditional ZNN models. Hence, in this subsection, a varying parameter is added to FTCZNN model (16) for solving time-varying linear matrix inequalities, and the varying parameter FTCZNN model can be directly given as

$$
\dot{E}(t)=-\left(t^{\gamma}+\gamma\right) \operatorname{STP}(E(0)) \diamond \Psi(E(t)),
$$

where $\gamma \geq 2, \operatorname{STP}(\cdot)$ is defined in formula (4), the operator $\diamond$ is defined in equation (5), and $\Psi(\cdot)$ is the sign-bi-power activation function which is defined in equation (12). 
Using the same example in Subsection 4.1, from Fig. 6, it can be seen that the varying-parameter FTCZNN model (57) with $\gamma=3$ has better performance in convergence rate, convergence time and robustness. In the future work, these features may be further researched and applied to practical engineering fields.

\section{Conclusions}

For the purpose of solving the time-varying LMIs more faster and in finite-time, three FTCZNN models have been presented and investigated in this work by exploring three nonlinear activation functions. It has been first proved that three FTCZNN models are globally stable according to the Lyapunov theory. Then, the convergence upper bounds of three FTCZNN models have been estimated to prove the finite-time convergence performance. Numerical comparison results ulteriorly reveal the superiority performance of three FTCZNN models for solving LMIs. That is to say, the state solutions by using FTCZNN models can converge to the theoretical solution set of time-varying LMIs accurately and rapidly. The future work may focus on the following two topics. One is the study and investigation of the application of time-varying linear matrix inequality in practice. The other is to develop and optimize the neural models to possess superior convergence performance.

\section{References}

[1] F. Xu, Z. Li, Z. Nie, et al., Zeroing neural network for solving timevarying linear equation and inequality systems, IEEE Transactions on Neural Networks and Learning Systems, 30 (8) (2019) 2346 - 2357.

[2] L. Jin, S. Li, J. Yu, et al., Robot manipulator control using neural networks: A survey, Neurocomputing, 285 (2018) 23-34.

[3] L. Xiao, B. Liao, S. Li, Z. Zhang, L. Ding, and L. Jin, Design and analysis of FTZNN applied to the real-time solution of a nonstationary Lyapunov equation and tracking control of a wheeled mobile manipulator, IEEE Transactions on Industrial Informatics, 14(1) (2018) 98-105.

[4] P. Witczak, K. Patan, M. Witczak, et al., A neural network approach to simultaneous state and actuator fault estimation under unknown input decoupling, Neurocomputing, 250 (9) (2017) 65-75. 
[5] B. Huang, G. Hui, D. Gong, et al., A projection neural network with mixed delays for solving linear variational inequality, Neurocomputing, 125 (125) (2014) 28-32.

[6] L. Xiao, B. Liao, S. Li, and K. Chen, Nonlinear recurrent neural networks for finite-time solution of general time-varying linear matrix equations, Neural Networks, 98 (2018) 102-113.

[7] L. Xiao, S. Li, F. Lin, Z. Tan, A.H. Khan, Zeroing neural dynamics for control design: Comprehensive analysis on stability, robustness, and convergence speed, IEEE Transactions on Industrial Informatics, 15(5) (2019) 2605-2616.

[8] K. M. Grigoriadis, R. E. Skelton, Low-order control design for LMI problems using alternating projection methods, Automatica, 32 (8) (1996) 1117-1125.

[9] S. Limanond, J. Si, Neural network-based control design: an LMI approach, IEEE Transactions on Neural Networks, 9 (6) (1998) 1422-1429.

[10] Z. Zhang, T. Zheng, Global asymptotic stability of periodic solutions for delayed complex-valued Cohen Grossberg neural networks by combining coincidence degree theory with LMI method, Neurocomputing, 289 (2018) 220-230.

[11] S. Lakshmanan, C. Lim, M. Prakash, et al., Neutral-type of delayed inertial neural networks and their stability analysis using the LMI approach, Neurocomputing, 230 (2017) 243-250.

[12] L. Xiao, Z. Zhang, Z. Zhang, W. Li, and S. Li, Design, verification and robotic application of a novel recurrent neural network for computing dynamic Sylvester equation, Neural Networks, 105 (2018) 185-196.

[13] Z. Quan, L. Huang, S. Yu, et al., Novel LMI-based condition on global asymptotic stability for BAM neural networks with reaction-diffusion terms and distributed delays, Neurocomputing, 136 (2014) 213-223.

[14] L. Xiao, Y. Zhang, J. Dai, K. Chen, S. Yang, W. Li, B. Lin, L. Ding, and J. Li, A new noise-tolerant and predefined-time ZNN model for timedependent matrix inversion, Neural Networks, 117 (2019) 124-134. 
[15] X. Li, J. Shen, LMI approach for stationary oscillation of interval neural networks with discrete and distributed time-varying delays under impulsive perturbations, IEEE Transactions on Neural Networks, 21 (10) (2010) 1555-1563.

[16] L. Xiao, S. Li, K. Li, L. Jin, and B. Liao, Co-design of finite-time convergence and noise suppression: a unified neural model for time varying linear equations with robotic applications, IEEE Trans. Syst., Man, Cybern., Syst., (2019) doi: 10.1109/TSMC.2018.2870489.

[17] W. Yu, J. Cao, W. Lu, Synchronization control of switched linearly coupled neural networks with delay, Neurocomputing, 73 (4-6) (2010) 858-866.

[18] D. Guo, Y. Zhang, A new inequality-based obstacle-avoidance MVN scheme and its application to redundant robot manipulators, IEEE Transactions on Systems Man and Cybernetics Part C, 42 (6) (2012) 1326-1340.

[19] Y. Zhang, J. Wang, Obstacle avoidance for kinematically redundant manipulators using a dual neural network, IEEE Transcations on Cybernetics, 34 (1) (2004) 752-759.

[20] L. Xiao, Y. Zhang, Z. Hu, and J. Dai, Performance benefits of robust nonlinear zeroing neural network for finding accurate solution of lyapunov equation in presence of various noises, IEEE Transactions on Industrial Informatics, 15 (9) (2019) 5161-5171.

[21] F. Cheng, T. Chen, Y. Sun, Resolving manipulator redundancy under inequality constraints, IEEE Transactions on Robotics and Automation, 10 (1) (1994) 65-71.

[22] Y. Zhang, B. Cai, L. Zhang, et al., Bi-criteria velocity minimization of robot manipulators using a linear variational inequalities-based primaldual neural network and PUMA560 example, Advanced Robotics, 22 (1314) (2008) 1479-1496.

[23] Y. Zhang, M. Yang, H. Huang, et al., New discrete solution model for solving future different-level linear inequality and equality with robot manipulator control, IEEE Transactions on Industrial Informatics, 15 (4) (2019) 1975-1984. 
[24] A. Luzi, A. Fradkov, J. Biannic, et al., Structured adaptive control for solving LMIs, IFAC Proceedings Volumes, 46 (11) (2013) 426-431.

[25] X. Hu, Dynamic system methods for solving mixed linear matrix inequalities and linear vector inequalities and equalities, Applied Mathematics and Computation, 216 (4) (2010) 1181-1193.

[26] C. Lin, C. Lai , T. Huang, A neural network for linear matrix inequality problems, IEEE Transactions on Neural Networks, 11 (5) (2000) 10781092.

[27] L. Xiao, Y. Zhang, Different Zhang functions resulting in different ZNN models demonstrated via time-varying linear matrix-vector inequalities solving, Neurocomputing, 121 (2013) 140-149.

[28] D. Guo, Y. Zhang, Zhang neural network for online solution of timevarying linear matrix inequality aided with an equality conversion, IEEE Transactions on Neural Networks and Learning Systems, 25 (2) (2014) 370-382.

[29] D. Guo, Y. Zhang, ZNN for solving online time-varying linear matrixvector inequality via equality conversion, Applied Mathematics and Computation, 259 (2015) 327-338.

[30] M. Ali, Stability of Markovian jumping recurrent neural networks with discrete and distributed time-varying delays, Neurocomputing, 149 (2015) 1280-1285.

[31] L. Jin, S. Li, B. Liao, et al., Zeroing neural networks: A survey, Neurocomputing, 267 (2017) 597-604.

[32] L. Xiao, Y. Zhang, Zhang neural network versus gradient neural network for solving time-varying linear inequalities, IEEE Transactions on Neural Networks, 22 (10) (2011) 1676-1684.

[33] L. Xiao, Y. Zhang, Two new types of Zhang neural networks solving systems of time-varying nonlinear inequalities, IEEE Transactions on Circuits and Systems I, 59 (10) (2012) 2363-2373.

[34] F. Yu, L. Liu, L. Xiao, et al., A robust and fixed-time zeroing neural dynamics for computing time-variant nonlinear equation using a novel nonlinear activation function, Neurocomputing, 350 (20) (2019) 108-116. 
[35] L. Xiao, S. Li, J. Yang, et al., A new recurrent neural network with noise-tolerance and finite-time convergence for dynamic quadratic minimization, Neurocomputing, 285 (12) (2018) 125-132.

[36] Z. Zhang, L. Zheng, J. Weng, et al., A new varying-parameter recurrent neural-network for online solution of time-varying Sylvester equation, IEEE Transactions on Cybernetics, 48 (11) (2018) 3135-3148.

[37] Z. Zhang, Y. Lu, L. Zheng, et al., A new varying-parameter convergentdifferential neural-network for solving time-varying convex QP problem constrained by linear-equality, IEEE Transactions on Automatic Control, 63 (12) (2018) 4110-4125.

[38] Z. Zhang, T. Fu, Z. Yan, et al., A varying-parameter convergentdifferential neural network for solving joint-angular-drift problems of redundant robot manipulators. IEEE/ASME Transactions on Mechatronics, 23 (2) (2018) 679-689.

[39] Z. Zhang, L. Zheng, A complex varying-parameter convergentdifferential neural-network for solving online time-varying complex Sylvester equation, IEEE Transactions on Cybernetics, 49 (10) (2019) 3627-3639.

[40] Y. Zhang, S. S. Ge, Design and analysis of a general recurrent neural network model for time-varying matrix inversion, IEEE Transactions on Neural Networks, 16 (6) (2005) 1477-1490.

[41] Y. Zhang, C. Yi, D. Guo, et al., Comparison on gradient-based neural dynamics and Zhang neural dynamics for online solution of nonlinear equations, International Symposium on Intelligence Computation and Applications, 20 (1) (2011) 1-7.

[42] D. P. Bertsekas, J. N. Tsitsiklis, Neuro-Dynamic Programming, 1996, Athena.

[43] J. Kamruzzaman, S. M. Aziz, A note on activation function in multilayer feedforward learning, in: IEEE International Joint Conference on Neural Networks, 2002, pp. 519-523. 